\title{
The PI3K Pathway: Background and Treatment Approaches
}

\author{
Michael P. Lux Peter A. Fasching Michael G. Schrauder Alexander Hein Sebastian M. Jud \\ Claudia Rauh Matthias W. Beckmann
}

University Breast Center for Franconia, Department of Gynecology, Erlangen University Hospital, CCC Erlangen-EMN, Erlangen, Germany

\section{Keywords}

Breast cancer - Estrogen receptor - Endocrine resistance . PI3K - Phosphatidylinositol 3-kinase - PIK3CA .

Phosphatidylinositol-4,5-bisphosphate 3-kinase catalytic subunit alpha

\section{Summary}

Two-thirds of all breast cancer patients with metastases have a hormone receptor (HR)-positive/human epidermal growth factor receptor 2 (HER2)-negative subtype. Endocrine therapy is the treatment of choice in these patients since in addition to its effectiveness it can also maintain the patients' quality of life over a longer term. However, 44-62\% of postmenopausal patients with metastatic breast carcinoma have primary tamoxifen resistance. After $3-5$ years, $30-40 \%$ of the patients receiving tamoxifen treatment develop secondary resistance. Understanding the way in which resistance develops is therefore essential for developing treatment approaches that can prevent or reverse endocrine resistance. The phosphatidylinositol 3-kinase (PI3K)/Akt/mammalian target of rapamycin (mTOR) signaling pathway plays a central role here. As a result of the numerous interactions involved, complex issues arise that need to be taken into account in the development and use of therapeutic agents. In addition, this signaling pathway is the one that most frequently undergoes mutations in breast cancer. The prognostic and predictive significance of individual mutations has not yet been fully explained, but it might provide a basis for patient selection in clinical studies. Initial research results on the use of PI3K inhibitors suggest that this may be a highly promising therapeutic approach, with an acceptable side effect profile.

(c) 2016 S. Karger GmbH, Freiburg

\section{Introduction}

Although the prognosis in patients with the luminal A/B subtype is basically better than in those with triple-negative or human epidermal growth factor receptor 2 (HER2)-positive breast carcinoma, patients with hormone receptor (HR)-positive/HER2-negative breast cancer represent two-thirds of all breast cancer patients with metastases [1]. Endocrine therapy is the first-line treatment option for metastatic HR-positive breast carcinoma [2]. In general, endocrine therapy is preferable since, in addition to being effective, it also maintains the patient's quality of life, in comparison with chemotherapy.

Endocrine resistance is a major problem, and a distinction is made between primary and secondary resistance. A significant proportion of patients have de novo or primary resistance, or they develop endocrine resistance during the course of treatment. Only 50\% of the patients with metastatic estrogen receptor (ER)-positive breast cancer benefit from first-line endocrine therapy, and all of those who respond initially acquire resistance, with tumor relapse and growth [3]. With secondary resistance as well, the progression-free survival (PFS) times with additional endocrine treatment options are short.

Therefore, major goals are to understand the way in which endocrine resistance arises and to develop therapeutic agents that can reverse it or prevent it from developing. In this context, the focus is on the phosphatidylinositol 3-kinase (PI3K)/Akt/mammalian target of rapamycin (mTOR) signaling pathway. This is the most frequently mutated signaling pathway in breast carcinoma. Both experimental and clinical data support the hypothesis that, in addition to mitogen-activated protein kinase (MAPK/ERK), the pathway plays a significant role in endocrine resistance.

\section{The PI3K/Akt/mTOR Signaling Pathway}

$\mathrm{PI} 3 \mathrm{~K} / \mathrm{Akt} / \mathrm{mTOR}$ is an intracellular signaling pathway that responds to hormones and growth factors. Its activation leads to nu-

\section{KARGER}

(c) 2016 S. Karger GmbH, Freiburg

Fax +497614520714 
Fig. 1. Overview of the PI3K/Akt/mTOR pathway (reproduced from [9], with permission). RTK = receptor tyrosine kinase, $\mathrm{P}=$ phosphate, $\mathrm{IRS} 1=$ insulin receptor substrate- $1, \mathrm{PIP}_{2}=$ phosphatidylinositol 4,5-bisphosphate, $\mathrm{PIP}_{3}=$ phosphatidylinositol 3,4,5-trisphosphate, $\mathrm{PI} 3 \mathrm{~K}=$ phosphatidylinositol 3-kinase, $\mathrm{Akt}=$ activated protein kinase $\mathrm{B}, \mathrm{mTOR}=$ mammalian target of rapamycin, rictor $=$ rapamycin-insensitive companion of $\mathrm{mTOR}$, raptor $=$ regulatory-associated protein of $\mathrm{mTOR}, \mathrm{TSC}=$ tuberous sclerosis complex, Rheb = Ras homolog enriched in brain, GDP = guanosine diphosphate, GTP = guanosine triphosphate, $\mathrm{S} 6 \mathrm{~K} 1=$ ribosomal $\mathrm{S6}$ kinase $1,4 \mathrm{EBP} 1=4 \mathrm{E}$-binding protein 1 , eIF4e $=$ eukaryotic translation initiation factor $4 \mathrm{E}$.

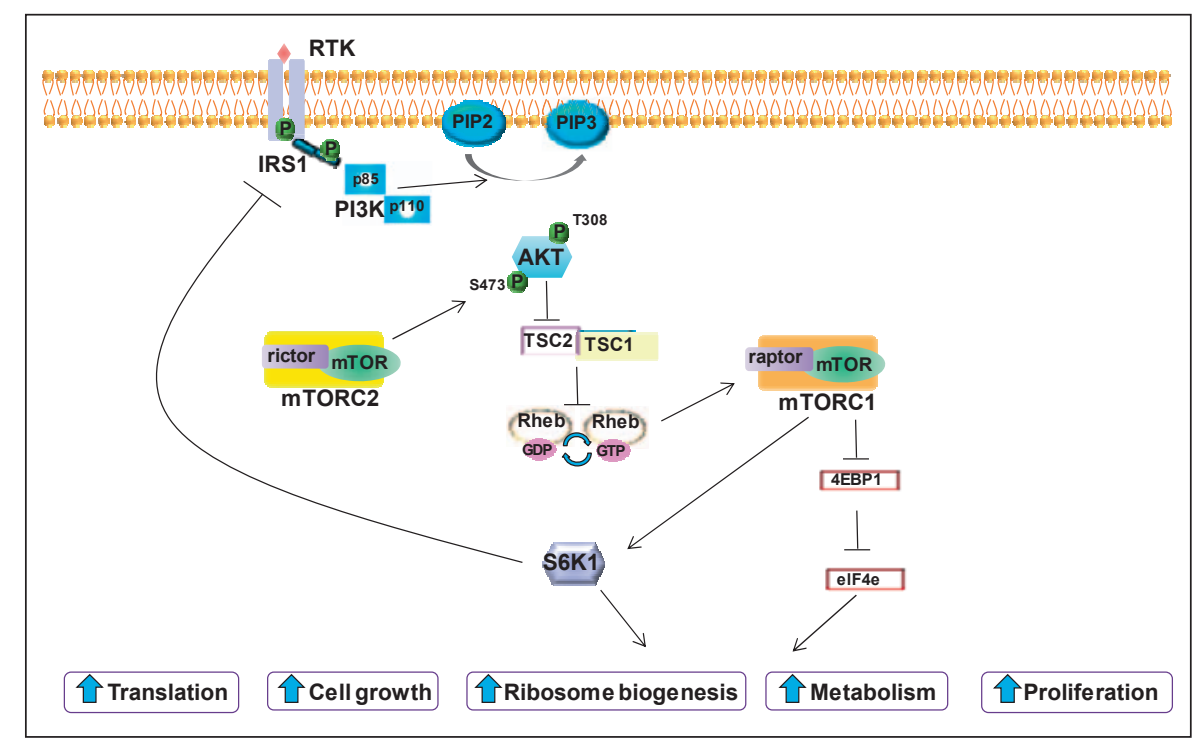

merous cellular processes such as increased cell growth, cell proliferation, and cellular motility, a shift toward glycolytic metabolism, and also increased cell migration and deregulated apoptosis [4].

PI3Ks are grouped into 3 classes (I-III) based on their structure and substrate specificity [5]. The PI3K heterodimer belonging to class IA plays a central role in the signaling pathway [6]. The heterodimer consists of 2 subunits. The regulatory subunit, p85, controls the activation of the catalytic subunit, p110, depending on the stimulation of growth receptor tyrosine kinases [7]. Subunits $\mathrm{p} 110 \alpha, \mathrm{p} 110 \beta$, and $\mathrm{p} 110 \delta$ are encoded by PIK3CA, PIK3CB, and $P I K 3 C D$, while the regulatory subunit is encoded by PIK3R1, PIK3R2, and PIK3R3 [8]. p110a and p110 $\beta$ are ubiquitously expressed. Class IB consists of $\mathrm{p} 110 \gamma$, which is encoded by PIK3CG. Expression of $\mathrm{p} 110 \delta$ and $\mathrm{p} 110 \gamma$ is generally limited to hematopoietic and immunological cells.

Activation takes place through the binding of various extracellular growth factors (e.g., epidermal growth factor (EGF), insulinlike growth factor 1 (IGF-1), and insulin) to their membranebound tyrosine kinase receptors (fig. 1) [9]. The respective receptor dimerizes, is autophosphorylated and recruits adaptor proteins such as insulin receptor substrate (IRS) 1 and IRS2. Subsequent phosphorylation of phosphatidylinositol-4,5-bisphosphate $\left(\mathrm{PIP}_{2}\right)$ into phosphatidylinositol-3,4,4-trisphosphate $\left(\mathrm{PIP}_{3}\right)$ leads to the phosphorylation of Akt, a serine/threonine kinase that further influences the tumor cell cycle for growth and survival (fig. 1) [10].

PTEN (phosphatase and tensin homolog deleted on chromosome 10) is an important tumor suppressor that has a contrary effect and dephosphorylates $\mathrm{PIP}_{3}$ to $\mathrm{PIP}_{2}$. PIP 3 levels are thus directly regulated by the opposing effects of PI3K and PTEN.

mTOR is a serine/threonine kinase that is downstream from PI3K and Akt. Two different complexes involving mTOR, mTORC1 and mTORC2, have different functions. mTORC1 is activated by inhibition of tumor suppressor tuberous sclerosis $1 / 2$ (TSC1/2) and subsequently influences the cell metabolism, leading to anabolic cell growth.
Activation of mTORC1 also leads to a negative feedback loop [9]. Direct inhibition of mTORC1 by an administered treatment can suspend the negative feedback loop, leading to the paradoxical activation of the signaling pathway and expression of multiple receptor tyrosine kinases such as HER2 and the IGF-1 receptor (IGF1R) [11]. Both this paradoxical activation and the activation of alternative signaling pathways need to be taken into account in the context of therapeutic approaches.

\section{The PI3K Signaling Pathway and Endocrine Resistance}

Deregulation of the class 1A PI3K signaling pathway is associated with the development of resistance to numerous forms of treatment, including antiestrogens, trastuzumab, radiotherapy, and chemotherapy [12]. It has been demonstrated experimentally that $\mathrm{PI} 3 \mathrm{~K}$ activation is associated with both de novo and also acquired endocrine resistance.

Preclinical data have confirmed that there is crosstalk between the ER and the PI3K/Akt/mTOR signaling pathway. Studies using breast carcinoma cell lines have shown an association between the activity of the Akt signaling pathway and endocrine resistance. Subsequently, an association was demonstrated between the activation of Akt or reduced PTEN expression and endocrine resistance in patients with metastatic breast carcinoma or recurrences after tamoxifen therapy $[13,14]$. Inhibition of Akt via the PI3K signaling pathway may in turn restore sensitivity to tamoxifen, aromatase inhibitors, and also fulvestrant [9].

Activation of the PI3K signaling pathway can be measured using the phosphorylated secondary products. A gene expression signature for PI3K activation based on the levels of phosphoprotein markers (P-AKT, P-p70S6K) is increased in HR-positive carcinomas, particularly in luminal $\mathrm{B}$ breast carcinomas. This suggests that luminal B tumors have higher levels of PI3K activity that may lead to a reduced response to antiestrogen therapy $[3,15]$. 
Table 1. Alterations in the PI3K signaling pathway (adapted from $[12,16])$

\begin{tabular}{|c|c|c|c|c|c|c|}
\hline \multirow[t]{2}{*}{ Gene } & \multirow[t]{2}{*}{ Aberration } & \multirow[t]{2}{*}{ Effect on signaling } & \multicolumn{3}{|l|}{ Frequency } & \multirow[t]{2}{*}{ Prognosis } \\
\hline & & & $\begin{array}{l}\text { HR-positive/ } \\
\text { luminal }\end{array}$ & $\begin{array}{l}\text { HER2- } \\
\text { positive }\end{array}$ & $\begin{array}{l}\text { TNBC/ } \\
\text { basal-like }\end{array}$ & \\
\hline ERB2 & $\begin{array}{l}\text { gene amplification } \\
\text { or overexpression }\end{array}$ & $\begin{array}{l}\text { hyperactivation of Erb2 } \\
\text { signaling (PI3K, MEK) }\end{array}$ & 10 & 100 & 0 & $\begin{array}{l}\text { correlates with } \\
\text { poorer outcome }\end{array}$ \\
\hline PTEN & $\begin{array}{l}\text { loss of function/ } \\
\text { reduced expression }\end{array}$ & $\begin{array}{l}\text { hyperactivation of PI3K } \\
\text { signaling }\end{array}$ & $29-44$ & 22 & 67 & $\begin{array}{l}\text { no consistent } \\
\text { correlation }\end{array}$ \\
\hline PIK3CA & activating mutation & $\begin{array}{l}\text { hyperactivation of PI3K } \\
\text { signaling }\end{array}$ & $28-47$ & $23-33$ & 8 & $\begin{array}{l}\text { ER+/HER2-, } \\
\text { better outcome; } \\
\text { ER+/HER2+, } \\
\text { poorer outcome }\end{array}$ \\
\hline PIK3CB & amplification & unknown & & & & unknown \\
\hline $\begin{array}{l}I G F 1 R \\
I N S R\end{array}$ & receptor activation & $\begin{array}{l}\text { activates IGF-1R/InsR } \\
\text { signaling (PI3K, MEK) }\end{array}$ & $41-48$ & $18-64$ & 42 & unknown \\
\hline FGFR1 & amplification & $\begin{array}{l}\text { hyperactivation of FGFR } \\
\text { signaling (PI3K, MEK) }\end{array}$ & $8.6-11.6$ & 5.4 & 5.6 & $\begin{array}{l}\text { correlates with } \\
\text { shorter RFS }\end{array}$ \\
\hline INPP4B & $\begin{array}{l}\text { reduced expression } \\
\text { or genomic loss }\end{array}$ & $\begin{array}{l}\text { hyperactivation of PI3K } \\
\text { signaling }\end{array}$ & 8 & 38 & 88 & $\begin{array}{l}\text { correlates with } \\
\text { poorer outcome }\end{array}$ \\
\hline$A K T 1$ & activating mutation & $\begin{array}{l}\text { hyperactivation of Akt } \\
\text { signaling }\end{array}$ & $2.6-3.8$ & 0 & 0 & unknown \\
\hline AKT2 & amplification & $\begin{array}{l}\text { hyperactivation of Akt } \\
\text { signaling }\end{array}$ & 2.8 & & & unknown \\
\hline
\end{tabular}

EGFR $=$ Epidermal growth factor receptor, ER = estrogen receptor, FGFR $=$ fibroblast growth factor receptor, HER2 = human epidermal growth factor receptor 2, HR = hormone receptor, IGF-1R = insulin-like growth factor-1 receptor, InsR = insulin receptor, MEK = MAPK/ERK kinase, $\mathrm{mTORC1}=$ mammalian target of rapamycin complex 1, PI3K = phosphatidylinositol 3-kinase, $\mathrm{RFS}=$ relapse-free survival, $\mathrm{TNBC}=$ triple-negative breast cancer .

\section{Mutations in the PI3K Signaling Pathway and Their Prognostic and Predictive Values}

An overview of aberrations in the PI3K signaling pathway is presented in table 1 . Loss of the PTEN gene and mutations in the PIK3CA gene are some of the most frequent aberrations seen in malignancies such as breast cancer [6]. It is estimated that the $\mathrm{PI} 3 \mathrm{~K} / \mathrm{Akt} / \mathrm{mTOR}$ signaling pathway is activated in $70 \%$ of all breast carcinomas as a result of genetic alterations [16].

\section{PIK3CA}

The highest frequency of mutations in PIK3CA, which codes for the catalytic subunit $\mathrm{p} 110 \mathrm{a}$, is found in ER-positive tumors and particularly in the luminal A subtype (luminal A, 45\%; luminal B, 29\%) [6]. A meta-analysis including 26 studies demonstrated a significant association between PIK3CA mutations and ER and progesterone receptor (PR) expression (odds ratio (OR) 1.92, 95\% confidence interval (CI) 1.65-2.23; OR 1.88, 95\% CI 1.61-2.20, respectively) [17]. Although these mutations often occur in ER-positive breast carcinomas, their clinical relevance and their prognostic and predictive value are still matters of debate.

Up to $80 \%$ of the PIK3CA mutations occur in hotspots in the helical (E542K and E545K in exon 9) and kinase (H1047R or H1047L in exon 20) domains of p110a [12]. Exon 9 mutations are considered to enable p110a to escape the inhibitory effect of p 85 via the Src homology 2 (SH2) domain [5]. These mutations increase the PI3K activity and induce cellular transformation in vitro and tumorigenicity in vivo when overexpressed in human mammary epithelial cells. They also induce mammary tumor formation in transgenic mice $[10,12$, 18]. However, PIK3CA mutations have not been found to be associated with increased phosphorylation of Akt, S6, or 4EBP1, which actually represent markers for an activated PI3K signaling pathway. Overall, the data are still inconsistent and might also be explained by a lack of standardization in the measurement of phosphorylated proteins. Moreover, the acquisition of mutations in PIK3CA does not appear to be responsible for the development of endocrine resistance. Arthur et al. [19] have profiled matched primary and recurrent samples from 120 breast cancer patients treated with endocrine therapy. $43 \%$ of the patients had at least 1 PIK3CA mutation at diagnosis and $41 \%$ at the time of recurrence. Only $8 \%$ of the patients changed their PIK3CA mutation status (4 gains, 2 losses) with local recurrence, metastatic disease, or progression on primary endocrine treatment.

In retrospective studies, PIK3CA mutations have been found to be associated with a good long-term outcome in primary ER-positive tumors [20]. A meta-analysis including 5 studies noted a significant correlation between PIK3CA mutations and a better recurrence-free survival (hazard ratio (HR) $0.76,95 \%$ CI 0.59-0.98; $\mathrm{P}=$ 0.03 ) [17]. Another meta-analysis including 6 studies found a similar result in relation to disease-free survival (HR 0.72, 95\% CI 0.57$0.91 ; \mathrm{P}=0.006)$ [21]. A further study analyzed the association between PIK3CA mutations and outcome in 4,540 postmenopausal patients with ER-positive breast cancer who received endocrine therapy in the context of the TEAM trial, a multinational, randomized phase III trial comparing exemestane with tamoxifen for 
the first 2.5-3 years, followed by exemestane [22]. PIK3CA mutations were frequent (at $39.8 \%$ ) and most of the mutations were detected in the sequences coding for the helical or kinase domains (E542K 13.5\%, E545K 21.9\%, H1047R 44.3\%). Patients with PIK3CA mutations were at significantly lower risk of distant metastases after 5 years (distant relapse-free survival $91 \%$ vs. $88 \%$, HR 0.76, 95\% CI $0.63-0.91 ; \mathrm{P}=0.003$ ), but the multivariate analysis failed to show that the PIK3CA mutation status was an independent prognostic marker. The authors also examined the effects of mutations in the helical versus kinase domains. The influence of these different mutations on the outcome is still a matter of controversy. No statistically significant differences were observed between helical and kinase domain mutations in the study (HR 0.82, 95\% CI 0.60-1.14; P = 0.24).

Overall, PIK3CA mutation and increased ER positivity can thus be regarded as favorable prognostic parameters. However, these considerations are mainly related to ER-positive and HER2-negative carcinomas in the context of endocrine therapy. Several publications have shown that, in HER2-positive breast carcinomas, the PI3KCA mutation represents a negative factor for the outcome of treatment with trastuzumab. A retrospective study with tumor samples from 240 patients with HER2-positive early breast carcinoma who were treated with epirubicin, 5-fluorouracil, and cyclophosphamide followed by 1 year of trastuzumab noted reduced overall survival in the presence of the mutation in comparison with the wild type (multivariate $\mathrm{HR} 2.14,95 \% \mathrm{CI} 1.01-4.51 ; \mathrm{P}=0.046$ ) [23]. In relation to the metastatic situation, a retrospective study including 139 patients with HER2-positive tumors and treatment including trastuzumab found that PTEN loss and PIK3CA mutation represented significant independent parameters associated with a shorter time to progression and reduced survival [24]. However, it is still questionable whether the PIK3CA mutation represents a purely prognostic factor in HER2-positive tumors or whether it may also be predictive for anti-HER2 therapy, since most retrospective studies have not included a control arm without anti-HER2 therapy. In the framework of the FinHER study, in which patients received either trastuzumab or chemotherapy alone for 9 weeks, the PIK3CA mutation status was not found to be predictive of a benefit from trastuzumab [25].

In summary, it may be noted that PIK3CA mutations:

- occur most often in ER-positive and HER2-negative tumors;

- may coexist with additional PI3K-activating mechanisms, such as HER2 amplification or PTEN loss;

- may potentially represent a favorable prognostic parameter in the luminal subtypes; and

- may be a predictive factor for a poorer outcome under trastuzumab-based therapies [5].

\section{PTEN}

PTEN is a tumor suppressor that deactivates the PI3K signaling pathway. PTEN loss often coexists with PIK3CA mutations [5]. Switching-off of PTEN and also overexpression of oncogenes that activate the PI3K/Akt signaling pathway are associated with endocrine resistance to tamoxifen and fulvestrant [26]. Loss of PTEN gene expression in HR-positive carcinomas is also predictive of reduced recurrence-free survival after tamoxifen [27]. However, re-
Table 2. Overview of PI3K inhibitors

\begin{tabular}{ll}
\hline Class & Substance \\
\hline Pan-PI3K inhibitors & BKM120 (buparlisib) \\
& XL-147 \\
& PX-866 \\
& PKI-587 \\
& GDC-0941 \\
p110a-specific & wortmannin \\
inhibitors & LY294002 \\
& BYL719 (alpelisib) \\
& GDC-0032 \\
p1108-specific & INK-1117 \\
inhibitors & CAL-101 \\
Dual PI3K/mTOR & BEZ235 \\
inhibitors & BGT226 \\
& PF-4691502 \\
& GDC-0980 \\
& XL-765 \\
\hline
\end{tabular}

duced PTEN expression is more often observed in triple-negative breast carcinomas (67\% vs. $29-44 \%$ in ER-positive tumors) [28]. Tumor cells with PTEN loss tend to depend more on PIK3CB than on PIK3CA. A pan-PI3K inhibitor might be considered preferable here over a selective p110 inhibitor.

\section{INPP4B}

Inositol polyphosphate 4-phosphatase type IIB (INPP4B) is another potential biomarker. It is a tumor suppressor that regulates $\mathrm{PI} 3 \mathrm{~K} /$ Akt and is involved in the dephosphorylation of $\mathrm{PIP}_{3}$ to $\mathrm{PIP}_{2}$. Its deletion correlates with poor prognosis. Patients with an ERpositive/INPP4B-deficient tumor show reduced survival in comparison with patients with an ER-positive/INPP4B-positive tumor [29]. INPP4B deficiency might be a candidate for the application of PI3K inhibitors [6].

\section{Therapeutic Approaches}

The importance of the PI3K signaling pathway in relation to endocrine resistance and also the high frequency of alterations in the pathway in breast carcinoma make it an interesting therapeutic target. Multiple inhibitors of the PI3K/Akt/mTOR signaling pathway are currently in the process of preclinical development or have already been investigated in clinical studies. At present, more than 100 studies listed on ClinicalTrial.gov investigate the use of PI3K inhibitors in breast carcinoma. A distinction is made between panPI3K inhibitors, p110a-specific inhibitors, p110 $\delta$-specific inhibitors, and dual PI3K/mTOR inhibitors (table 2). It is as yet unclear whether pan-PI3K inhibitors or isoform-selective inhibitors represent the more effective approach.

As the ER persists in most ER-positive breast carcinomas with resistance to endocrine therapy and a response to estrogens is possible, PI3K inhibitors are combined with an antiestrogen therapy. Somatic aberrations such as PIK3CA and AKT1 mutations, PTEN and INPP4B loss, and PIK3CB and AKT2 amplification may indi- 
cate either disturbed activation or dependency on the PI3K signaling pathway. On the basis of the existing data, it is assumed that PIK3CA mutations and PTEN loss increase the sensitivity to PI3K/ Akt inhibitors. The PIK3CA mutation H1047R, for example, represents an independent predictor for response [30]. This should be taken into account in the selection of patients for studies with PI3K inhibitors; due to the heterogeneous state of the data, the ideal biomarker has not yet been identified.

\section{p110 $\alpha$-Specific Inhibitors}

The starting point for $\mathrm{p} 110 \mathrm{a}$-specific inhibitors was the fungal metabolite wortmannin, which leads to powerful and irreversible inhibition of PI3K. The target is the p110 subunit. The inhibition of cell growth was demonstrated in tumor cell lines [31]. The first PI3K inhibitor to be developed was LY294002, which had the same mode of action as wortmannin. In preclinical studies, enhancement of cytotoxic therapies was demonstrated in various tumor entities [32]. PX866 is a synthetic derivative of wortmannin. Due to an irreversible interaction with the adenosine triphosphate (ATP) binding site, it is a potent PI3K inhibitor. Highly promising results have already been achieved with it in combination with docetaxel in solid tumors [33].

BYL719 (alpelisib) is an oral, class I $\alpha$-specific PI3K inhibitor. In vitro, BYL719 has been shown to inhibit the proliferation of cell lines harboring PIK3CA mutations. In vivo, BYL719 demonstrated dose-dependent inhibition of tumor growth in various subcutaneous tumor transplant models. BYL719 was subsequently tested in a phase I study in patients with solid tumors who had a PIK3CA mutation. A favorable safety profile was reported. Partial remissions were observed in 7 of 39 patients, 2 of whom had ER-positive breast carcinomas. At a dosage of $270 \mathrm{mg} /$ day, 15 patients with ERpositive and HER2-negative breast carcinomas achieved a PFS period of 5.5 months [28].

Combination of alpelisib or a placebo with fulvestrant is currently being investigated in a phase III study in postmenopausal women and in men with HR-positive, HER2-negative advanced breast carcinoma and progression after treatment with an aromatase inhibitor (the SOLAR-1 study) [34]. The effectiveness of BYL719 in comparison with buparlisib or a placebo in combination with letrozole is also being analyzed in a randomized, double-blind study in the neoadjuvant setting. The primary end-point is the assessment of the pathologic complete response ( $\mathrm{pCR}$ ) rate during neoadjuvant treatment in the 2 cohorts (PIK3CA-mutated and PIK3CA wild-type tumors).

Another agent is currently being evaluated in the framework of the SANDPIPER study [35]. In this phase III study, postmenopausal patients with ER-positive, HER2-negative locally advanced or metastatic breast carcinomas with progression during or after aromatase inhibitor therapy receive treatment with the PI3K inhibitor taselisib (GDC-0032) and fulvestrant, or a placebo and fulvestrant (2:1 randomization).

\section{Pan-PI3K Inhibitors}

Initial data on pan-PI3K inhibitors from randomized studies were obtained from the phase III study FERGI, which investigated the addition of pictilisib (GDC-0941; $340 \mathrm{mg} /$ day), a class I PI3K inhibitor, to fulvestrant (500 mg, q28d). A total of 168 patients with ER-positive advanced breast carcinoma who had progressed during aromatase inhibitor therapy were included [36]. The initial results showed a non-significant increase in PFS from 5.1 to 6.6 months (HR 0.74; $\mathrm{P}=0.096$ ). In this study, the presence of a PI3KCA mutation did not have any predictive value.

Buparlisib (BKM120) is an oral pan-class I PI3K inhibitor that blocks all 4 isoforms of PI3K $(\alpha, \beta, \gamma, \delta)$ [37]. In a phase Ib study with buparlisib in combination with letrozole in patients with ERpositive, HER2-negative metastatic breast carcinoma ( $\mathrm{n}=46$ with evaluable disease), complete remission and partial remission were observed, as well as stable disease in 25 patients [38]. A correlation with the PIK3CA mutation was not identified in the study.

Additional administration of buparlisib in patients with breast carcinoma is currently being analyzed using the BELLE study approaches. The BELLE-2 study is the first randomized phase III study to investigate the safety and efficacy of buparlisib in combination with fulvestrant. A total of 1,147 postmenopausal patients with HRpositive and HER2-negative locally advanced or metastatic breast carcinoma with progression during or after aromatase inhibitor treatment were randomly assigned (1:1) to receive buparlisib (100 $\mathrm{mg} /$ day) and fulvestrant (500 $\mathrm{mg}$ ) or a placebo [39]. The primary end-points of the study are the PFS in the overall group and also in the PI3K-activated group (with PIK3CA mutation and/or PTEN loss). In all, $53.1 \%$ and $52.7 \%$ of the patients, respectively, had 1 course of endocrine therapy in the metastatic situation and $19.4 \%$ and $22.4 \%$, respectively, had 2 or more courses of endocrine treatment. In the overall group, treatment with buparlisib and fulvestrant was associated with a statistically significant prolongation of PFS by 1.9 months in comparison with placebo and fulvestrant treatment (6.9 vs. 5.0 months; HR $0.79,95 \%$ CI $0.67-0.89$; P < 0.01). In the PI3K-activated group, the PFS in the experimental arm was 6.8 months and that in the control arm was 4.0 months (HR 0.76, 95\% CI $0.60-0.97 ; \mathrm{P}=0.014$ ). Data from an exploratory analysis of patients with a PIK3CA mutation in circulating tumor DNA (ctDNA; $\mathrm{n}=200$ ) are interesting. In those with a confirmed mutation, a PFS of 7.0 months was achieved in comparison with 3.2 months in the placebo arm (HR 0.56, 95\% CI 0.39-0.80; P $<0.001$ ). The authors concluded that clinically significant efficacy is achieved, particularly in the subgroup with a PIK3CA mutation in ctDNA. In addition, it might be possible to identify a predictive factor for efficacy using a blood sample. The overall survival data are still awaited and will be analyzed after 588 cases of death in the overall group.

The appropriate treatment after combination of an mTOR inhibitor with an aromatase inhibitor represents another clinical challenge. The BELLE-3 study is evaluating the option of treatment with BKM120 in combination with fulvestrant in patients who have progressed after treatment with an aromatase inhibitor and an mTOR inhibitor. Due to the association between resistance to mTOR inhibition and activation of the PI3K/Akt/mTOR signaling pathway, it is assumed that all tumors have an activated PI3K signaling pathway [34].

The BELLE-4 study is a phase II study investigating a combination with weekly administration of paclitaxel. 


\section{Dual PI3K/mTOR Inhibitors}

The TORC1 inhibitor everolimus is able to eliminate the negative feedback to PI3K activators. On the one hand, this supports the hypothesis that direct inhibition of PI3K is effective while, on the other hand, it has led to the development of dual kinase inhibitors that act on both mTOR and PI3K (e.g., XL765). SAR245409, a $\mathrm{PI} 3 \mathrm{~K} / \mathrm{mTOR}$ inhibitor, is being evaluated in a phase I/II study in combination with letrozole.

PI3K Inhibitors in Combination with Other Therapeutic Agents As inhibition of PI3K or Akt leads in turn to the up-regulation/ activation of numerous tyrosine kinases, combination of PI3K/ Akt/TORC1 inhibitors with tyrosine kinase inhibitors may be considered in order to achieve an optimal antitumor effect.

Preclinical and clinical data make it clear that ER-positive tumor cells that are treated with tamoxifen are able to evade the ER blockade via HER2 overexpression [40]. Combined blockade of the ER and HER2 signaling pathways may therefore represent an effective treatment approach.

Synergistic effects between BKM120 and trastuzumab have been demonstrated in HER2-positive cell lines. It was also possible to restore sensitivity to trastuzumab in trastuzumab-resistant xenografts. In a phase I/II study, combined treatment with BKM120 and trastuzumab in patients with trastuzumab-resistant HER2positive breast carcinomas showed good tolerability, with a clinical benefit of $75 \% .17 \%$ of the patients had partial remissions and $58 \%$ had stable disease after 6 weeks or longer [41].

BKM120 has already been investigated in the neoadjuvant setting in combination with trastuzumab. In the NeoPHOEBE study, patients with HER2-positive primary breast carcinomas of $>2 \mathrm{~cm}$ were stratified in accordance with their PIK3CA mutation status and randomly assigned after central testing to receive neoadjuvant therapy with 6 weeks of trastuzumab and buparlisib followed by 12 weeks of trastuzumab plus paclitaxel plus buparlisib, or a placebo [42]. The primary end-point was pCR at the time of surgery (ypT0/ is). A total of 50 patients were included. Recruitment subsequently had to be stopped due to toxicities and early discontinuations of treatment, as well as an inability to reach the primary study aim. The pCR rates did not differ significantly between the treatments, either overall or relative to the stratified subgroups. The overall response rate (ORR) after week 6 also did not differ between the treatments, although there was a trend toward a better ORR with buparlisib in the ER-positive subgroup $(\mathrm{P}=0.053$; interaction between buparlisib and ER status, $\mathrm{P}=0.032$ ). Buparlisib led to a decrease in Ki-67 from baseline to day 15 in all patients and in the ER-positive subgroup. The authors recommend further investigation of the addition of a PI3K inhibitor to anti-HER2 therapy in the ER-positive and HER2-positive group.
In another study, BKM120 is being investigated in combination with lapatinib in patients with advanced trastuzumab-resistant HER2-positive breast carcinoma and PIK3CA or PTEN alterations. Preclinical data suggest that cyclin-dependent kinase 4/6 (CDK4/6) inhibition may also have synergistic effects with PI3K inhibitors, in order to reduce the cell survival capacity and overcome intrinsic or secondary endocrine resistance [43]. Phase I/II studies are thus currently investigating a combination of the 2 inhibitors, as in the combination of BYL719 or BKM120 with LEE011 (ribociclib) and fulvestrant or letrozole in postmenopausal women with advanced HR-positive breast carcinomas [16].

\section{Side Effect Profile of PI3K Inhibitors}

The PI3K inhibitors are presenting oncologists with a novel side effect profile. According to the phase I studies that have been conducted, the most frequent side effects include gastrointestinal toxicities (diarrhea, nausea, vomiting), skin rashes, hyperglycemia, and also mood changes - which may occur in 1 in 5 patients and may range from anxiety and depression to hallucinations and affective disturbances.

This was also seen in the results most recently presented from the BELLE-3 study mentioned above [39]. Grade III adverse events occurred in $63.2 \%$ of cases, and grade IV events, in $14.1 \%(27.4 \%$ and $4.6 \%$, respectively, in the control arm). The most frequent adverse events seen included increased transaminases, hyperglycemia, skin rashes, and also anxiety and depression $(22.3 \%$ and $26.2 \%$, respectively, for the latter in all grades).

The psychological side effects are probably a result of PI3K inhibition in the central nervous system, as the inhibitors are able to cross the blood-brain barrier. This in turn might offer a potential approach to the treatment of brain metastases. According to the study data, these side effects can be controlled through dose reduction, with or without antidepressant agents or anxiolytics [41].

\section{Conclusions}

Endocrine resistance in patients with HR-positive breast carcinoma continues to represent an oncological challenge. Alterations in the PI3K signaling pathway are among the most frequent somatic mutations in ER-positive breast carcinomas, which are associated with endocrine resistance. Numerous PI3K inhibitors are currently undergoing preclinical and clinical development. The data available so far indicate highly promising results.

\section{Disclosure Statement}

The authors are investigators in ongoing trials with BYL719 and BKM120.

\section{References}

1 Tumorregister München: Spezielle Auswertungen Mammakarzinom (C50): Metastasierte Patientinnen. www.tumorregistermuenchen.de/facts/spec/spec_C50f 08_20151021_met_hr.pdf, 2013 [last accessed November 13, 2015].
2 Kommission Mamma der Arbeitsgemeinschaft Gynäkologische Onkologie e.V.: Diagnostik und Therapie primärer und metastasierter Mammakarzinome. Version 2016.1D. www.ago-online.de.
3 Fu X, Osborne CK, Schiff R: Biology and therapeutic potential of PI3K signaling in ER+/HER2-negative breast cancer. Breast 2013;22(suppl 2):S12-S18. 
4 Banerji S, Cibulskis K, Rangel-Escareno C, Brown KK, Carter SL, Frederick AM, et al.: Sequence analysis of mutations and translocations across breast cancer subtypes. Nature 2012;486:405-409.

5 Mukohara T: PI3K mutations in breast cancer: prognostic and therapeutic implications. Breast Cancer (Dove Med Press) 2015;7:111-123.

6 Paplomata E, O’Regan R: The PI3K/AKT/mTOR pathway in breast cancer: targets, trials and biomarkers. Ther Adv Med Oncol 2014;6:154-166.

7 Cantley LC: The phosphoinositide 3-kinase pathway. Science 2002;296:1655-1657.

8 Engelman JA, Luo J, Cantley LC: The evolution of phosphatidylinositol 3-kinases as regulators of growth and metabolism. Nat Rev Genet 2006;7:606-619.

9 Hasson SP, Rubinek T, Ryvo L, Wolf I: Endocrine resistance in breast cancer: focus on the phosphatidylinositol 3-kinase/Akt/mammalian target of rapamycin signaling pathway. Breast Care 2013;8:248-255.

10 Zhao L, Vogt PK: Class I PI3K in oncogenic cellular transformation. Oncogene 2008;27:5486-5496.

11 Chandarlapaty S, Sawai A, Scaltriti M, Rodrik-Outmezguine V, Grbovic-Huezo O, Serra V, Majumder PK, Baselga J, Rosen N: Akt inhibition relieves feedback suppression of receptor tyrosine kinase expression and activity. Cancer Cell 2011;19:58-71.

12 Miller TW, Balko JM, Arteaga CL: Phosphatidylinositol 3-kinase and antiestrogen resistance in breast cancer. J Clin Oncol 2011;29:4452-4461.

13 Tokunaga E, Kimura Y, Mashino K, Oki E, Kataoka A, Ohno S, Morita M, Kakeji Y, Baba H, Maehara Y: Activation of PI3K/Akt signaling and hormone resistance in breast cancer. Breast Cancer 2006;13:137-144.

14 Shoman N, Klassen S, McFadden A, Bickis MG, Torlakovic E, Chibbar R: Reduced PTEN expression predicts relapse in patients with breast carcinoma treated by tamoxifen. Mod Pathol 2005;18:250-259.

15 Sørlie T, Perou CM, Tibshirani R, Aas T, Geisler S, Johnsen H, Hastie T, Eisen MB, van de Rijn M, Jeffrey SS, Thorsen T, Quist H, Matese JC, Brown PO, Botstein D, Lønning PE, Børresen-Dale AL: Gene expression patterns of breast carcinomas distinguish tumor subclasses with clinical implications. Proc Natl Acad Sci USA 2001;98:10869-10874.

16 Lee JJ, Loh K, Yap Y-S: PI3K/Akt/mTOR inhibitors in breast cancer. Cancer Biol Med 2015;12:342-354.

17 Pang B, Cheng S, Sun S-P, An C, Liu Z-Y, Feng X, Liu G-J: Prognostic role of PIK3CA mutations and their association with hormone receptor expression in breast cancer: a meta-analysis. Sci Rep 2014;4:6255.

18 Meyer DS, Brinkhaus H, Müller U, Müller M, Cardiff RD, Bentires-Alj M: Luminal expression of PIK3CA mutant H1047R in the mammary gland induces heterogeneous tumors. Cancer Res 2011;71:4344-4351.

19 Arthur LM, Turnbull AK, Renshaw L, Keys J, Thomas JS, Wilson TR, Lackner MR, Sims AH, Dixon JM: Changes in PIK3CA mutation status are not associated with recurrence, metastatic disease or progression in endocrine-treated breast cancer. Breast Cancer Res Treat 2014;147:211-219.

20 Loi S, Haibe-Kains B, Majjaj S, Lallemand F, Durbecq V, Larsimont D, Gonzalez-Angulo AM, Pusztai L, Symmans WF, Bardelli A, Ellis P, Tutt AN, Gillett CE, Hennessy BT, Mills GB, Phillips WA, Piccart MJ, Speed TP, McArthur GA, Sotiriou C: PIK3CA mutations associated with gene signature of low mTORC1 signaling and better outcomes in estrogen receptorpositive breast cancer. Proc Natl Acad Sci USA 2010; 107:10208-10213.

-21 Liu Y-R, Jiang Y-Z, Zuo W-J, Yu K-D, Shao Z-M: PIK3CA mutations define favorable prognostic biomarkers in operable breast cancer: a systematic review and meta-analysis. Onco Targets Ther 2014;7:543-552.
22 Sabine VS, Crozier C, Brookes CL, Drake C, Piper T, van de Velde CJ, Hasenburg A, Kieback DG, Markopoulos C, Dirix L, Seynaeve C, Rea DW, Bartlett JM: Mutational analysis of PI3K/Akt signaling pathway in tamoxifen exemestane adjuvant multinational pathology study. J Clin Oncol 2014;32:2951-2958.

23 Jensen JD, Knoop A, Laenkholm AV, Grauslund M, Jensen MB, Santoni-Rugiu E, Andersson M, Ewertz M: PIK3CA mutations, PTEN, and pHER2 expression and impact on outcome in HER2-positive early-stage breast cancer patients treated with adjuvant chemotherapy and trastuzumab. Ann Oncol 2012;23:20342042.

24 Razis E, Bobos M, Kotoula V, Eleftheraki AG, Kalofonos HP, Pavlakis K, Papakostas P, Aravantinos G, Rigakos G, Efstratiou I, Petraki K, Bafaloukos D, Kostopoulos I, Pectasides D, Kalogeras KT, Skarlos D, Fountzilas G: Evaluation of the association of PIK3CA mutations and PTEN loss with efficacy of trastuzumab therapy in metastatic breast cancer. Breast Cancer Res Treat 2011;128:447-456.

25 Loi S, Michiels S, Lambrechts D, Fumagalli D, Claes B, Kellokumpu-Lehtinen PL, Bono P, Kataja V, Piccart MJ, Joensuu H, Sotiriou C: Somatic mutation profiling and associations with prognosis and trastuzumab benefit in early breast cancer. J Natl Cancer Inst 2013;105: 960-967.

26 Shou J, Massarweh S, Osborne CK, Wakeling AE, Ali S, Weiss H, Schiff R: Mechanisms of tamoxifen resistance: increased estrogen receptor-HER2/neu cross-talk in ER/HER2-positive breast cancer. J Natl Cancer Inst 2004;96:926-935.

27 Saal LH, Johansson P, Holm K, Gruvberger-Saal SK, She QB, Maurer M, Koujak S, Ferrando AA, Malmström P, Memeo L, Isola J, Bendahl PO, Rosen N, Hibshoosh H, Ringnér M, Borg A, Parsons R: Poor prognosis in carcinoma is associated with a gene expression signature of aberrant PTEN tumor suppressor pathway activity. Proc Natl Acad Sci USA 2007;104:7564-7569.

28 Gonzalez-Angulo AM, Ferrer-Lozano J, Stemke-Hale K, Sahin A, Liu S, Barrera JA, Burgues O, Lluch AM, Chen H, Hortobagyi GN, Mills GB, Meric-Bernstam F: PI3K pathway mutations and PTEN levels in primary and metastatic breast cancer. Mol Cancer Ther 2011; 10:1093-1101.

29 Gewinner C, Wang ZC, Richardson A, Teruya-Feldstein J, Etemadmoghadam D, Bowtell D, Barretina J, Lin WM, Rameh L, Salmena L, Pandolfi PP, Cantley LC: Evidence that inositol polyphosphate 4-phosphatase type II is a tumor suppressor that inhibits PI3K signaling. Cancer Cell 2009;16:115-125.

30 Janku F, Tsimberidou AM, Garrido-Laguna I, Wang X, Luthra R, Hong DS, Naing A, Falchook GS, Moroney JW, Piha-Paul SA, Wheler JJ, Moulder SL, Fu S, Kurzrock R: PIK3CA mutations in patients with advanced cancers treated with PI3K/Akt/mTOR axis inhibitors. Mol Cancer Ther 2011;10:558-565.

31 Powis G, Hill SR, Frew TJ, Sherrill KW: Inhibitors of phospholipid intracellular signaling as antiproliferative agents. Med Res Rev 1995;15:121-138.

$32 \mathrm{Ng} \mathrm{SSW}$, Tsao MS, Chow S, Hedley DW: Inhibition of phosphatidylinositide 3-kinase enhances gemcitabineinduced apoptosis in human pancreatic cancer cells. Cancer Res 2000;60:5451-5455.

33 Bowles DW, Ma WW, Senzer N, Brahmer JR, Adjei AA, Davies M, Lazar AJ, Vo A, Peterson S, Walker L, Hausman D, Rudin CM, Jimeno A: A multicenter phase 1 study of PX-866 in combination with docetaxel in patients with advanced solid tumours. Br J Cancer 2013;109:1085-1092.
34 Massacesi C, Di Tomaso E, Urban P, Germa C, Quadt C, Trandafir L, Aimone P, Fretault N, Dharan B, Tavorath R, Hirawat S: PI3K inhibitors as new cancer therapeutics: implications for clinical trial design. Onco Targets Ther 2016;9:203-210.

35 Baselga J, Cortes J, De Laurentiis M, Dieras V, Harbeck N, Hsu JY, et al.: SANDPIPER: Phase III study of the PI3-kinase (PI3K) inhibitor taselisib (GDC-0032) plus fulvestrant in patients (pts) with estrogen receptor (ER)-positive, HER2-negative locally advanced or metastatic breast cancer (BC) enriched for pts with PIK3CA-mutant tumors. J Clin Oncol 2016;34(suppl): abstr TP617.

36 Krop I, Johnston S, Mayer IA, Dickler M, Ganju V, Forero-Torres A, et al.: The FERGI phase II study of the PI3K inhibitor pictilisib (GDC-0941) plus fulvestrant vs fulvestrant plus placebo in patients with ER+ aromatase inhibitor (AI)-resistant advanced or metastatic breast cancer - Part I results. San Antonio Breast Cancer Symp 2014;S2-2.

37 Maira SM, Pecchi S, Huang A, Burger M, Knapp M, Sterker D, Schnell C, Guthy D, Nagel T, Wiesmann M, Brachmann S, Fritsch C, Dorsch M, Chène P, Shoemaker K, De Pover A, Menezes D, Martiny-Baron G, Fabbro D, Wilson CJ, Schlegel R, Hofmann F, GarcíaEcheverría C, Sellers WR, Voliva CF: Identification and characterization of NVP-BKM120, an orally available pan-class I PI3-kinase inhibitor. Mol Cancer Ther 2012;11:317-328.

38 Mayer IA, Abramson VG, Isakoff SJ, Forero A, Balko JM, Kuba MG, Sanders ME, Yap JT, Van den Abbeele AD, Li Y, Cantley LC, Winer E, Arteaga CL: Stand up to cancer phase Ib study of pan-phosphoinositide3-kinase inhibitor buparlisib with letrozole in estrogen receptor-positive/human epidermal growth factor receptor 2-negative metastatic breast cancer. J Clin Oncol 2014;32:1202-1209.

39 Baselga J, Im S, Iwata H, Clemons M, Ito Y, Awada A, et al.: PIK3CA status in circulating tumor DNA (ctDNA) predicts efficacy of buparlisib (BUP) plus fulvestrant (FULV) in postmenopausal women with endocrine-resistant HR+/HER2- advanced breast cancer (BC): first results from the randomized, phase III BELLE-2 trial. San Antonio Breast Cancer Symp 2015; S6-1.

40 Arpino G, Wiechmann L, Osborne CK, Schiff R: Crosstalk between the estrogen receptor and the HER tyrosine kinase receptor family: molecular mechanism and clinical implications for endocrine therapy resistance. Endocr Rev 2008;29:217-233.

41 Saura C, Bendell J, Jerusalem G, Su S, Ru Q, De Buck S, Mills D, Ruquet S, Bosch A, Urruticoechea A, Beck JT, Di Tomaso E, Sternberg DW, Massacesi C, Hirawat S, Dirix L, Baselga J: Phase Ib study of buparlisib plus trastuzumab in patients with HER2-positive advanced or metastatic breast cancer that has progressed on trastuzumab-based therapy. Clin Cancer Res 2014;20: 1935-1945.

42 Loibl S, de la Pena L, Nekljudova V, Zardavas D, Michiels S, Denkert C, et al.: Phase II, randomized, parallel-cohort study of neoadjuvant buparlisib (BKM120) in combination with trastuzumab and paclitaxel in women with HER2-positive, PIK3CA mutant and PIK3CA wild-type primary breast cancer NeoPHOEBE. San Antonio Breast Cancer Symp 2015. P1-14-1.

43 Vora SR, Juric D, Kim N, Mino-Kenudson M, Huynh T, Costa C, Lockerman EL, Pollack SF, Liu M, Li X, Lehar J, Wiesmann M, Wartmann M, Chen Y, Cao ZA, Pinzon-Ortiz M, Kim S, Schlegel R, Huang A, Engelman JA: CDK 4/6 inhibitors sensitize PIK3CA mutant breast cancer to PI3K inhibitors. Cancer Cell 2014;26:136-149. 